\title{
Abnormal nocturnal blood pressure profile is associated with mild cognitive impairment in the elderly: the J-SHIPP study
}

\author{
Haiyan Guo ${ }^{1}$, Yasuharu Tabara ${ }^{2}$, Michiya Igase $^{1}$, Miyuki Yamamoto $^{1}$, Namiko Ochi $^{1}$, Tomoko Kido ${ }^{1}$, \\ Eri Uetani ${ }^{1}$, Keiko Taguchi ${ }^{1}$, Tetsuro Miki ${ }^{1}$ and Katsuhiko Kohara ${ }^{1}$
}

Mild cognitive impairment $(\mathrm{MCl})$, a syndrome characteristic of the transition phase between normal cognitive function and dementia, has been shown to carry the risk of progression to dementia. Dysregulation of blood pressure (BP) is thought to be an indicator of cerebrovascular damage, including cognitive impairment. Here, we investigated the possible association of circadian BP variation with $\mathrm{MCl}$ in community-dwelling persons exhibiting no definitive dementia. Our study enrolled 144 persons ( $68 \pm 7$ years). Nocturnal BP profile was defined as dipper, with a $10-19 \%$ drop in nocturnal systolic BP; extreme dipper, $\geqslant 20 \%$ drop; non-dipper, $0-10 \%$ drop; and riser, any increase in nocturnal $\mathrm{BP}$. $\mathrm{MCl}$ was assessed using the $\mathrm{MCl}$ screen, a cross-validated, staff-administered battery of tests. Subjects with $\mathrm{MCl}(n=38)$ were significantly older $(74 \pm 6,67 \pm 6$ years, $P<0.001)$ and had higher frequency of apolipoprotein $\mathrm{E} \varepsilon 4$ allele $(36.8,18.9 \%, P=0.018)$. Although the ambulatory measured $\mathrm{BP}$ and the percent changes in nocturnal systolic $\mathrm{BP}(-10 \pm 12 \%$ and $-12 \pm 8 \%$, respectively; $P=0.291)$ did not differ between $\mathrm{MCl}$ subjects and normal controls, frequency of $\mathrm{MCl}$ was significantly higher in the extreme dippers $(32.0 \%)$, non-dippers (30.0\%) and risers (50.0\%) than in dippers $(13.2 \%, P=0.018)$. Multiple logistic regression analysis identified a blunted nocturnal BP decline, non-dipping or increase in nocturnal BP and extreme drop in BP as potent determinants of $\mathrm{MCl}$ (odds ratio 3.062, $P=0.039$ ), after adjustment for possible confounding factors, including apolipoprotein $E \varepsilon 4$ genotype. Abnormal nocturnal BP profile was found to be a strong indicator of $\mathrm{MCl}$ in otherwise apparently healthy community-dwelling elderly persons.

Hypertension Research (2010) 33, 32-36; doi:10.1038/hr.2009.172; published online 23 October 2009

Keywords: ambulatory blood pressure monitoring; mild cognitive impairment; nocturnal blood pressure

\section{INTRODUCTION}

Mild cognitive impairment (MCI) is a syndrome defined as cognitive decline greater than expected for an individual's age and education level that does not notably interfere with activities of daily life. ${ }^{1} \mathrm{MCI}$ is classified into four subtypes: amnestic MCI involving only memory impairment (single domain) or memory impairment along with deficits in another cognitive domain such as language function, visuospatial skills, or executive function (multiple domain) and non-amnestic MCI involving discrete cognitive impairments in single or multiple domains other than memory function. ${ }^{2}$ MCI prevalence has been estimated to be between 6 and $10 \%$ based on community assessments, ${ }^{3}$ a frequency about four times that of dementia. MCI also exhibits Alzheimer-type dementia-like neuropathology on autopsy, and at least half of those diagnosed with MCI, in particular amnestic MCI subtype, eventually progress into Alzheimer-type dementia (AD) with a smaller percentage progression to other forms of dementia, such as vascular dementia, frontotemporal dementia or dementia with Lewy bodies. ${ }^{2}$ Early detection of amnestic MCI in the transition phase between healthy aging and dementia is essential in the secondary prevention of dementia. Although no disease-modifiable treatment specifically targeting degenerative dementia has been discovered, control of risk factors such as isolated systolic hypertension is known to be associated with a decrease in incidence of dementia. ${ }^{4}$

Blood pressure (BP) instability has been hypothesized to be associated with MCI due to the traditional cardiovascular risk factors common to both, including arterial stiffness, ${ }^{5}$ hypertension ${ }^{6}$ and diabetes mellitus. ${ }^{7}$ Matsubayashi et al. ${ }^{8}$ reported a significant association between postural dysregulation in systolic BP and low scores on neurobehavioral functional tests among elderly with no cognitive impairment. Few reports, however, have investigated the association between circadian $\mathrm{BP}$ variation and $\mathrm{MCI}$ in the general population.

${ }^{1}$ Department of Geriatric Medicine, Ehime University Graduate School of Medicine, Toon City, Ehime, Japan and ${ }^{2}$ Department of Basic Medical Research and Education, Ehime University Graduate School of Medicine, Toon City, Ehime, Japan

Correspondence: Dr Y Tabara, Department of Basic Medical Research and Education, Ehime University Graduate School of Medicine, Toon City, Ehime 791-0295, Japan. E-mail: tabara@m.ehime-u.ac.jp

Received 2 February 2009; revised 15 July 2009; accepted 30 August 2009; published online 23 October 2009 
Here, we analyzed the possible association between nocturnal BP profile and amnestic MCI in community-dwelling middle-aged to elderly general subjects with no definitive dementia.

\section{METHODS}

\section{Study subjects}

The study enrolled 144 middle-aged to elderly persons (aged $\geqslant 50$ years) with no definitive dementia (Table 1). Subjects were participants in the Ehime University Hospital medical checkup program, which is provided to residents of Ehime Prefecture, Japan, and is specifically designed to evaluate aging-related disorders such as atherosclerosis, cardiovascular disease and MCI. Among participants (aged $\geqslant 50$ years) from February 2006 to August 2008 (8 persons per week), who provided informed consent $(n=761)$, those who had no history of symptomatic cerebrovascular disease and definitive dementia $(n=759$, $99.7 \%)$, underwent MCI screening $(n=461,60.6 \%)$ and ambulatory BP monitoring $(n=239,31.4 \%)$, completed all clinical measurement and genotyping $(n=227,29.8 \%)$ and were not receiving antihypertensive treatment $(n=144,18.9 \%)$ were enrolled in the analysis. All clinical data used in this study were obtained through the checkup process. This cross-sectional investigation was carried out as part of the Shimanami Health Promoting Program (J-SHIPP Study), a longitudinal study evaluating factors related to cardiovascular disease, dementia and death. ${ }^{9}$ This series of studies was approved by the Ethics Committee of Ehime University Graduate School of Medicine.

\section{Assessment of MCI}

MCI was assessed by the Japanese version of the MCI screen (MCIS), ${ }^{10-12}$ a 10-min long, computationally scored, staff-administered test. Validity and specificity in the differentiation of normal aging from MCI and mild dementia are described elsewhere. The overall accuracy in discriminating both amnestic and mixed cognitive domain types of MCI from normal aging is $97 \% .{ }^{10}$ The MCIS has been translated into Japanese and cross validation has been confirmed using the Clinical Dementia Rating Scale as a reference (overall accuracy 96.4\%). ${ }^{12}$

\section{4-h ambulatory BP monitoring}

Ambulatory BP was monitored using a cuff-oscillometric device (TM-2431; A\&D, Tokyo, Japan), a fully automatic device that was pre-set to measure BP and heart rate every $15 \mathrm{~min}$ during the day (0700 to 2000 hours) and every 30 min at night. Outliers of ambulatory BP reading were rejected using an A\&D method; a most widely used simple rejection method that excludes BP readings outside of a specified range. ${ }^{13}$ Sleep duration was assessed by individual interview, and overall averages of 24 - $h$, awake and sleeping ambulatory BP values were calculated for each individual. The percentage decline in nocturnal $\mathrm{BP}$ was calculated with the following equation: ((daytime BP-nighttime $\mathrm{BP}) \times 100$ )/daytime BP. Subjects were classified into four groups based on nocturnal systolic BP decline: extreme dipper, with a decline in nocturnal systolic BP $\geqslant 20 \%$; dipper, $10-19 \%$; non-dipper, none to $9 \%$ and riser, increase in nocturnal systolic BP. ${ }^{14}$

\section{Measurement of arterial stiffness}

Brachial-ankle pulse wave velocity (baPWV) was measured as an index of arterial stiffness and calculated using the time interval between the wave fronts of the brachial and ankle waveforms and the path length from the brachium to the ankle. The path lengths from the suprasternal notch to the brachium (Lb) and ankle (La) were obtained using the following formulas: $\mathrm{Lb}=0.2195 \times$ height -2.0734 and $\mathrm{La}=0.8129 \times$ height +12.328 . A significant and strong correlation between the baPWV and carotid-femoral PWV has been reported. ${ }^{15} \mathrm{~A}$ brief explanation of this device as well as the validity and reproducibility of its measurements have been provided elsewhere. ${ }^{16}$

\section{Genotyping}

Genomic DNA was extracted from peripheral blood using a QIAamp DNA blood kit (Qiagen, Hilden, Germany). A single-nucleotide polymorphism (SNP; T334C [Cys112Arg], rs429358) on the apolipoprotein E (APOE) gene was analyzed using the TaqMan probe assay (Applied Biosystems, Foster City, CA, USA) with commercially available primers and probes purchased from the Assay-on-Demand system (C_3084793_20). The fluorescence level of PCR products was measured using an ABI PRISM 7900HT sequence detector (Applied Biosystems). The APOE isotype-related genotypes are combinations of the APOE $\varepsilon 2, \varepsilon 3$ and $\varepsilon 4$ alleles derived from the two genotypes of the rs429358 (T334C) and rs7412 (C472T): $\varepsilon 2,334 \mathrm{~T} / 472 \mathrm{~T} ; \varepsilon 3,334 \mathrm{~T} / 472 \mathrm{C}$ and $\varepsilon 4$, $334 \mathrm{C} / 472 \mathrm{C}$. Risk genotype for AD ( $\varepsilon 4)$ was detected by analyzing the SNP rs429358 (T334C). Frequency of homozygotes and heterozygotes of the $\varepsilon 4$

Table 1 Clinical characteristics of study subjects categorized by nocturnal BP profile

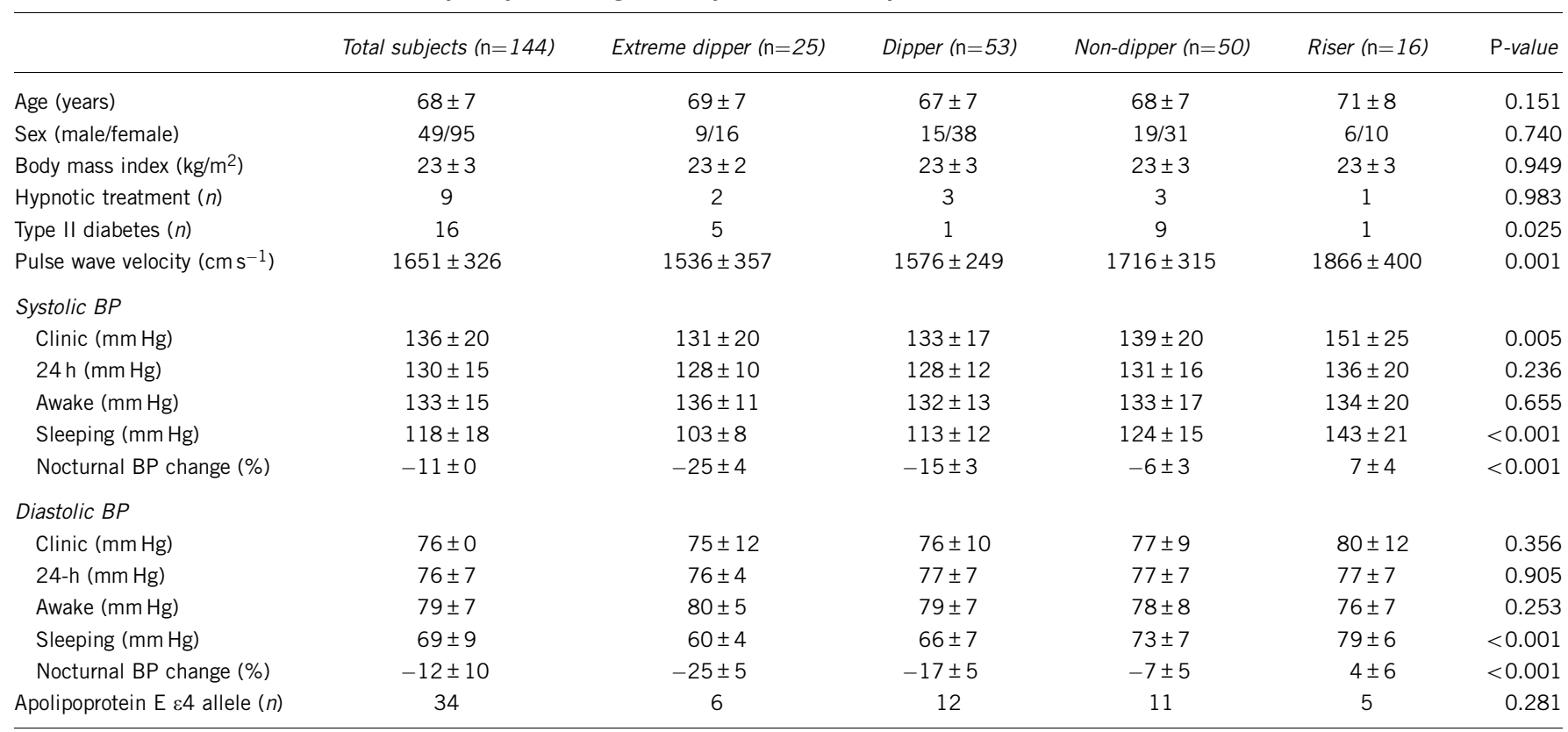

Abbreviation: BP, blood pressure.

Values are mean \pm s.d. Ambulatory blood pressure was measured in 15 -min intervals during the day ( 0700 to 2200 hours) and in 30 -min intervals at night. Circadian blood pressure pattern was defined as follows: extreme dipper, with a decline in nocturnal systolic blood pressure $\geqslant 20 \%$; dipper, $10-19 \%$; non-dipper, $0-9 \%$; and riser, increase in nocturnal systolic blood pressure. Type II diabetes was diagnosed as either or both fasting blood glucose $\geqslant 126 \mathrm{mg}$ per $100 \mathrm{ml}$ or the current use of oral antidiabetic agents. 
genotype in our subjects was $2.8 \%(n=4)$ and $20.8 \%(n=30)$, respectively ( $P$-value for Hardy-Weinberg equilibrium is 0.277 ).

\section{Statistical analysis}

Values are listed as mean \pm standard deviation. Analysis of variance was used to assess differences in numeric variables among groups, whereas the $\chi^{2}$-test was used to assess frequency differences. Factors independently associated with MCI were assessed by logistic regression analysis. All analyses were conducted using commercially available statistical software (JMP version 7, SAS Institute, Cary, NC, USA). A two-tailed $P<0.05$ was considered statistically significant.

\section{RESULTS}

Clinical characteristics of study subjects categorized by nocturnal BP profile are summarized in Table 1 . No significant differences were observed in age, sex or body mass index (BMI) among these four groups. However, frequency of type II diabetes was significantly higher in subjects with an abnormal nocturnal BP pattern (16.5\%) than in dippers $(1.9 \%, P=0.007)$. Non-dippers and risers also showed significantly higher clinic measured systolic BP and baPWV.

Clinical characteristics of the 38 subjects diagnosed with MCI are summarized in Table 2. A mini-mental state examination (MMSE) was administered to a small number of randomly selected subjects $(n=29)$, and subjects with MCI showed lower MMSE scores (mean score, $28.0 \pm 2.5)$ than the normal controls $(29.7 \pm 0.6)$. Further, MCI subjects were significantly older, more diabetic and more often had the APOE $\varepsilon 4$ genotype. MCI subjects also showed significantly higher ambulatory measured, but not clinic measured, systolic BP. Significant correlation was also observed between $\mathrm{MCI}$ and baPWV.

Although MCI subjects showed significantly higher average systolic BP over $24 \mathrm{~h}$, no marked differences were observed in the percent

Table 2 Clinical characteristics of study subjects with $\mathrm{MCI}$

\begin{tabular}{|c|c|c|c|}
\hline & $\begin{array}{l}\text { Mild cognitive } \\
\text { impairment } \\
\quad(\mathrm{n}=38)\end{array}$ & $\begin{array}{l}\text { Normal cognitive } \\
\quad \text { function } \\
(\mathrm{n}=106)\end{array}$ & P-value \\
\hline Age (years) & $74 \pm 6$ & $67 \pm 6$ & $<0.001$ \\
\hline Sex (male/female) & $17 / 21$ & $32 / 74$ & 0.104 \\
\hline Body mass index $\left(\mathrm{kg} / \mathrm{m}^{2}\right)$ & $23 \pm 3$ & $23 \pm 3$ & 0.818 \\
\hline Hypnotic treatment & 5 & 4 & 0.040 \\
\hline Type II diabetes $(n)$ & 7 & 9 & 0.095 \\
\hline $\begin{array}{l}\text { Brachial-ankle pulse } \\
\text { wave velocity }\left(\mathrm{cm} \mathrm{s}^{-1}\right)\end{array}$ & $1808 \pm 380$ & $1595 \pm 285$ & 0.001 \\
\hline \multicolumn{4}{|l|}{ Systolic BP } \\
\hline Clinic $(\mathrm{mm} \mathrm{Hg})$ & $139 \pm 19$ & $136 \pm 20$ & 0.358 \\
\hline 24-h (mm Hg) & $135 \pm 15$ & $128 \pm 14$ & 0.019 \\
\hline Awake $(\mathrm{mm} \mathrm{Hg})$ & $138 \pm 14$ & $131 \pm 15$ & 0.020 \\
\hline Sleeping (mm Hg) & $125 \pm 23$ & $116 \pm 15$ & 0.008 \\
\hline Nocturnal BP change (\%) & $-10 \pm 12$ & $-12 \pm 8$ & 0.291 \\
\hline \multicolumn{4}{|l|}{ Diastolic BP } \\
\hline Clinic $(\mathrm{mm} \mathrm{Hg})$ & $76 \pm 11$ & $77 \pm 10$ & 0.537 \\
\hline 24-h (mm Hg) & $76 \pm 6$ & $77 \pm 7$ & 0.888 \\
\hline Awake $(\mathrm{mm} \mathrm{Hg})$ & $78 \pm 6$ & $79 \pm 7$ & 0.774 \\
\hline Sleeping $(\mathrm{mm} \mathrm{Hg})$ & $70 \pm 10$ & $68 \pm 8$ & 0.193 \\
\hline Nocturnal BP change (\%) & $-10 \pm 12$ & $-13 \pm 9$ & 0.127 \\
\hline Apolipoprotein E $\varepsilon 4$ allele $(n)$ & 14 & 20 & 0.025 \\
\hline
\end{tabular}

Abbreviation: BP, blood pressure.

Values are mean \pm s.d. Mild cognitive impairment was assessed by $\mathrm{MCl}$ screen. ${ }^{12}$ Ambulatory blood pressure was measured in 15-min intervals during the day (0700 to 2000 hours) and at

30-min intervals at night. Type II diabetes was diagnosed as either or both fasting blood glucose $\geqslant 126 \mathrm{mg}$ per $100 \mathrm{ml}$ or the current use of oral antidiabetic agents. changes in nocturnal BP. However, the frequency of MCI was significantly higher and a J-shaped relationship was observed between these two parameters (Figure 1). Multiple logistic regression analysis for MCI was performed to further clarify the factors independently associated with MCI, including APOE $\varepsilon 4$ genotype and other possible confounding factors (Table 3). An abnormal nocturnal BP pattern was identified as an independent and potent determinant of MCI, with an odds ratio (3.062).

\section{DISCUSSION}

Here, we analyzed the possible association between nocturnal BP profile and amnestic MCI in community-dwelling middle-aged to elderly subjects with no definitive dementia. Results showed that both a blunted nocturnal BP decline, namely, non-dipping and increase in nocturnal BP, and extreme drop in BP were related to MCI; J-shaped relationship between nocturnal BP change and frequency of MCI. To our knowledge, this study is the first to identify a J-shaped relationship between nocturnal BP profile and MCI.

Although several attempts have been made to discern the mechanisms leading to a decline in cognitive performance in the elderly, most have been focused on cholinergic dysfunction, ${ }^{17}$ neurodegenerative disease, ${ }^{18,19}$ cerebrovascular disease $^{18}$ and mutations in APOE alleles. ${ }^{20}$ Arterial stiffness assessed by PWV has also been suggested to contribute to the progression of cognitive impairment. ${ }^{5}$ Here, however, we found that although the baPWV of MCI subjects was significantly higher than that of normal controls, the significance of this association was lost on adjustment for age. Further, BP was found not to be an independent determinant for MCI. The inverse relationship between $\mathrm{BP}$ and cognitive function previously found in longitudinal epidemiological studies may be unrecognizable in cross-sectional

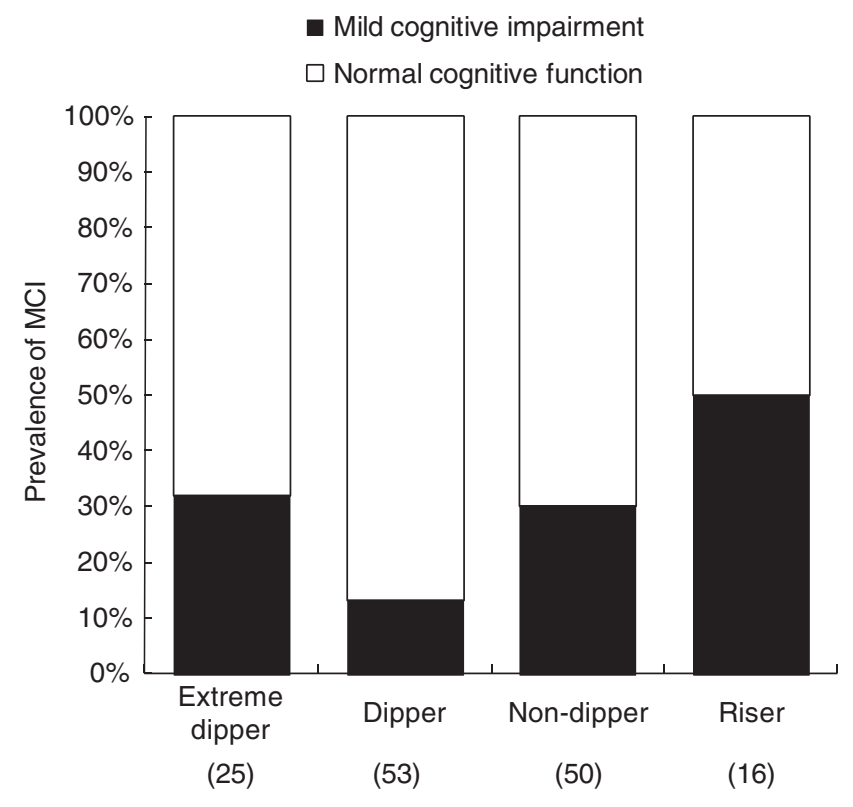

Figure 1 Frequency of mild cognitive impairment ( $\mathrm{MCl})$ subjects by nocturnal blood pressure (BP) pattern. Nocturnal BP pattern was defined as follows: extreme dipper, with a decline in nocturnal systolic BP $\geqslant 20 \%$; dipper, 10-19\%; non-dipper, 0-9\% and riser, increase in nocturnal systolic BP. The number of subjects in each group is represented in each column. Overall $P$-value assessed by $\chi^{2}$-test was 0.018 . 
Table 3 Multiple logistic regression analysis for $\mathrm{MCl}$

\begin{tabular}{lcr}
\hline & Odds ratio (95\% Cl) & \multicolumn{1}{c}{$\mathrm{P}$} \\
\hline Age (years) & $1.227(1.120-1.365)$ & $<0.001$ \\
Sex (female) & $0.350(0.122-0.951)$ & 0.039 \\
Clinic systolic BP (mm Hg) & $0.980(0.946-1.012)$ & 0.224 \\
Hypnotic treatment & $3.089(0.450-23.08)$ & 0.252 \\
Type II diabetes & $2.992(0.780-11.68)$ & 0.109 \\
Brachial-ankle pulse wave velocity $\left(\mathrm{cm} \mathrm{s}^{-1}\right)$ & $1.001(0.999-1.004)$ & 0.156 \\
Apolipoprotein E \&4 allele & $5.298(1.737-17.52)$ & 0.003 \\
Nocturnal BP profile dipper & Reference & \\
\multicolumn{1}{c}{ extreme dipper/ } & $3.062(1.057-9.930)$ & 0.039 \\
$\quad$ non-dipper/riser & & \\
\hline
\end{tabular}

Abbreviations: $\mathrm{BP}$, blood pressure; $\mathrm{Cl}$, confidence interval.

observational studies, presumably due to BP instability in the phases shortly before dementia, possibly related to a central dysregulation of prefrontal autonomic centers. ${ }^{21}$ Although we found no association between $\mathrm{MCI}$ and $\mathrm{BP}$ or baPWV, the prognostic significance of these parameters remains to be elucidated.

In contrast, we identified a marked association between abnormal nocturnal BP profile and MCI, an observation in agreement with the concept that early dementia is preceded by BP instability. An association has been reported between cognitive decline and increased BP variability as assessed by standard deviation of 24-h BP readings. ${ }^{22}$ These results lend support to our present finding that abnormalities in cognitive function are associated with a dysregulation in nocturnal BP profile.

However, the association of an extreme drop in nocturnal $\mathrm{BP}$ with cognitive decline remains to be determined. Among studies to date, van Boxtel et al. ${ }^{23}$ reported lower levels of cognitive function in non-dippers than in dippers, but did not separately analyze the effects of extreme dipping. Furthermore, Nagai et al. ${ }^{24}$ reported a positive association between the percent changes in nocturnal BP (as a numeric variable) and MMSE score in untreated hypertensive subjects, but again did not separately analyze the effects of an extreme decrease in BP. In this study, changes in nocturnal BP itself did not differ between MCI subjects and normal controls (Table 2) due to the high prevalence of MCI among not only non-dippers and risers but also extreme dippers (Figure 1). These discrepancies between the previous and present studies may have been partly due to differences in subject profile. However, extreme dippers are often given a poor prognosis in regard to the subsequent development of cerebrovascular disease ${ }^{25}$ and senile-onset depression ${ }^{26}$ that is frequently accompanied by cognitive decline. On this basis, both an extreme drop and a non-dipping pattern in nocturnal BP should be considered related to cognitive decline. Although the relationship between cerebral hypoperfusion and dementia has not been fully elucidated, ${ }^{27,28}$ lower nocturnal BP may cause dementia through cerebral hypoperfusion or may conversely be consequent to incipient dementia.

Underlying mechanisms relating abnormal nocturnal BP profile and MCI are uncertain. Sander et al. ${ }^{29}$ reported a positive association between abnormal nocturnal BP patterns with extent of cerebral white matter lesions in elderly persons. Kario et al. ${ }^{30}$ reported a higher frequency of asymptomatic lacunar infarction in elderly subjects with non-dipping profiles. These authors also found that reduced total brain matter was involved in the association between blunted nocturnal BP changes and lower MMSE score. ${ }^{24}$ Furthermore, in a recent longitudinal analysis found that increased white matter hyperintensities could be a predictor of progression from normal to MCI. ${ }^{31}$ Association of silent cerebral infarctions with the development of MCI was also reported in another community-based longitudinal study. ${ }^{32}$ By considering these findings together with our present data, asymptomatic cerebrovascular damages were thought to be one of possible mechanisms explainable for the relation between abnormal nocturnal BP profile and $\mathrm{MCI}$ in apparently healthy elderly persons.

Subjects of this study had no history of symptomatic cerebrovascular disease, and none were diagnosed as multiple domain-impaired amnestic MCI or non-amnestic MCI. As amnestic MCI eventually progress into $\mathrm{AD}$ with a smaller percentage progression to other forms of dementia, abnormal nocturnal BP profile may therefore predict future development of $\mathrm{AD}$ type dementia, but not vascular type dementia. $^{33}$

In this study, incidence of MCI was highest among risers. As a group, risers were slightly older and had higher baPWV. Among the others, non-dippers also showed high baPWV, whereas no marked difference was observed between extreme dippers and dippers (Table 1). The mechanism underlying the relationship between blunted nocturnal BP drop and MCI may thus differ somewhat with that of extreme dippers, that is, advanced arteriosclerosis and cerebral arterial remodeling in risers and non-dippers, ${ }^{34}$ and cerebral hypoperfusion due to the nocturnal BP fall in extreme dippers. ${ }^{28,35}$ Recently, excessive sympathetic activation was represented to be a factor potentially responsible for the day-night BP difference. ${ }^{36}$ Associations of salt sensitivity and sodium loadings with non-dipping profile were also reported. ${ }^{37-39}$ Furthermore, it was also reported that extreme dippers frequently complicate orthostatic hypertension. ${ }^{40}$ Autonomic dysfunction and consequent circadian BP dysreguration may represent a brain abnormality.

MCI is diagnosed as a level of cognitive deterioration not severe enough to consistently impair daily productivity and instrumental activities of daily living, but of sufficient severity to be noticeable by others and measurable by psychometric and other clinical assessments. ${ }^{41}$ Although MMSE is frequently used to assess cognitive function in clinical studies, its sensitivity and specificity in distinguishing MCI from normal aging has been reported as 71 and $85 \%$, respectively. ${ }^{10}$ In this study, however, MCIS had a higher accuracy than MMSE in differentiating MCI (overall accuracy in Japanese subjects is $96.4 \%){ }^{12}$ This observation further implies that an abnormal nocturnal BP profile may be a marker for distinguishing very early-stage cognitive decline.

Several limitations of this study warrant mention. First, subjects who underwent MCI screening were not randomly selected from among all medical checkup program participants, and thus the frequency of MCI among study subjects may not completely reflect prevalence in the general community. Second, our study samples are relatively small to identify risk factors with smaller effects. However, a nocturnal BP profile was identified as a risk factor for MCI in this study setting independent of established risk factors including APOE $\varepsilon 4$ genotype. Nocturnal BP profile therefore may have substantial power to identify at-risk persons. Third, we did not evaluate brain MRI images due to limited number of subjects who undertaken MRI measurement. We also did not measure neurological parameters. These data may provide further information to assess the clinical significance of nocturnal BP profile.

In conclusion, abnormal nocturnal BP profile was a sensitive marker of MCI in community-dwelling, apparently healthy elderly persons. Early detection of at-risk persons may lead to the prevention of cognitive function degeneration and the onset of dementia. 


\section{CONFLICT OF INTEREST}

The authors declare no conflict of interest.

\section{ACKNOWLEDGEMENTS}

We greatly appreciate the support of Tomoko Tanaka, Yoko Ochi and Chinatsu Hiraoka in clinical data collection and laboratory measurements. This study was supported by a Grant-in-Aid for Scientific Research from the Ministry of Education, Culture, Sports, Science and Technology of Japan, a Grant-in-Aid for Scientific Research from the Japan Arteriosclerosis Prevention Fund and a Research Promotion Award from Ehime University.

1 Gauthier S, Reisberg B, Zaudig M, Petersen RC, Ritchie K, Broich K, Belleville S, Brodaty $H$, Bennett D, Chertkow H, Cummings JL, de Leon M, Feldman H, Ganguli M, Hampel H, Scheltens P, Tierney MC, Whitehouse P, Winblad B. Mild cognitive impairment. Lancet 2006; 367: 1262-1270.

2 Marc E. Agronin. Alzheimer Disease and Other Dementias: A Practical Guide. Lippincott Williams \& Wilkins, Philadelphia, PA, 2007, pp 74-79.

3 DeCarli C. Mild cognitive impairment: prevalence, prognosis, aetiology, and treatment. Lancet Neurol 2003; 2: 15-21.

4 Forette F, Seux ML, Staessen JA, Thijs L, Birkenhäger WH, Babarskiene MR, Babeanu S, Bossini A, Gil-Extremera B, Girerd X, Laks T, Lilov E, Moisseyev V, Tuomilehto J, Vanhanen H, Webster J, Yodfat Y, Fagard R. Prevention of dementia in randomised double-blind placebo-controlled Systolic Hypertension in Europe (Syst-Eur) trial. Lancet 1998; 352: 1347-1351.

5 Hanon O, Haulon S, Lenoir H, Seux ML, Rigaud AS, Safar M, Girerd X, Forette F. Relationship between arterial stiffness and cognitive function in elderly subjects with complaints of memory loss. Stroke 2005; 36: 2193-2197.

6 Reitz C, Tang MX, Manly J, Mayeux R, Luchsinger JA. Hypertension and the risk of mild cognitive impairment. Arch Neurol 2007; 64: 1734-1740.

7 Luchsinger JA, Reitz C, Patel B, Tang MX, Manly JJ, Mayeux R. Relation of diabetes to mild cognitive impairment. Arch Neurol 2007; 64: 570-575.

8 Matsubayashi K, Okumiya K, Wada T, Osaki Y, Fujisawa M, Doi Y, Ozawa T. Postural dysregulation in systolic blood pressure is associated with worsened scoring on neurobehavioral function tests and leukoaraiosis in the older elderly living in a community. Stroke 1997; 28: 2169-2173.

9 Tabara Y, Igase M, Kido T, Ochi N, Miki T, Kohara K. Composition of lower extremity in relation to a high ankle-brachial index. J Hypertens 2009; 27: 167-173.

10 Shankle WR, Romney AK, Hara J, Fortier D, Dick MB, Chen JM, Chan T, Sun X. Methods to improve the detection of mild cognitive impairment. Proc Natl Acad Sci USA 2005; 102: 4919-4924.

11 Trenkle DL, Shankle WR, Azen SP. Detecting cognitive impairment in primary care: performance assessment of three screening instruments. J Alzheimers Dis 2007; 11: 323-335.

12 Cho A, Sugimura M, Nakano S, Yamada T. The Japanese MCI screen for early detection of Alzheimer's disease and related disorders. Am J Alzheimers Dis Other Demen 2008; 23: $162-166$.

13 Winnicki M, Canali C, Mormino P, Palatini P. Ambulatory blood pressure monitoring editing criteria: is standardization needed? Hypertension and Ambulatory Recording Venetia Study (HARVEST) Group, Italy. Am J Hypertens 1997; 10: 419-427.

14 Ohkubo T, Hozawa A, Yamaguchi J, Kikuya M, Ohmori K, Michimata M, Matsubara M, Hashimoto J, Hoshi H, Araki T, Tsuji I, Satoh H, Hisamichi S, Imai Y. Prognostic significance of the nocturnal decline in blood pressure in individuals with and without high 24-h blood pressure: the Ohasama study. J Hypertens 2002; 20: 2183-2189.

15 Sugawara J, Hayashi K, Yokoi T, Cortez-Cooper MY, DeVan AE, Anton MA, Tanaka H. Brachial-ankle pulse wave velocity: an index of central arterial stiffness? J Hum Hypertens 2005; 19: 401-406.

16 Yamashina A, Tomiyama H, Takeda K, Tsuda H, Arai T, Hirose K, Koji Y, Hori S, Yamamoto $Y$. Validity, reproducibility, and clinical significance of noninvasive brachialankle pulse wave velocity measurement. Hypertens Res 2002; 25: 359-364.

17 DeKosky ST, Ikonomovic MD, Styren SD, Beckett L, Wisniewski S, Bennett DA, Cochran EJ, Kordower JH, Mufson EJ. Upregulation of choline acetyltransferase activity in hippocampus and frontal cortex of elderly subjects with mild cognitive impairment. Ann Neurol 2002; 51: 145-155.

18 Bennett DA, Schneider JA, Bienias JL, Evans DA, Wilson RS. Mild cognitive impairment is related to Alzheimer disease pathology and cerebral infarctions. Neurology 2005; 64: 834-841.

19 Mufson EJ, Chen EY, Cochran EJ, Beckett LA, Bennett DA, Kordower JH. Entorhina cortex beta-amyloid load in individuals with mild cognitive impairment. Exp Neurol 1999; 158: 469-490.
20 Aggarwal NT, Wilson RS, Beck TL, Bienias JL, Berry-Kravis E, Bennett DA. The apolipoprotein E epsilon4 allele and incident Alzheimer's disease in persons with mild cognitive impairment. Neurocase 2005; 11: 3-7.

21 Birkenhäger WH, Forette F, Seux ML, Wang JG, Staessen JA. Blood pressure, cognitive functions, and prevention of dementias in older patients with hypertension. Arch Intern Med 2001; 161: 152-156.

22 Bellelli G, Pezzini A, Bianchetti A, Trabucchi M. Increased blood pressure variability may be associated with cognitive decline in hypertensive elderly subjects with no dementia. Arch Intern Med 2002; 162: 483-484.

23 van Boxtel MP, Henskens LH, Kroon AA, Hofman PA, Gronenschild EH, Jolles J, de Leeuw PW. Ambulatory blood pressure, asymptomatic cerebrovascular damage and cognitive function in essential hypertension. J Hum Hypertens 2006; 20: 5-13.

24 Nagai M, Hoshide S, Ishikawa J, Shimada K, Kario K. Ambulatory blood pressure as an independent determinant of brain atrophy and cognitive function in elderly hypertension. J Hypertens 2008; 26: 1636-1641.

25 Kario K, Pickering TG, Matsuo T, Hoshide S, Schwartz JE, Shimada K. Stroke prognosis and abnormal nocturnal blood pressure falls in older hypertensives. Hypertension 2001; 38: 852-857.

26 Hamada T, Murata T, Omori M, Takahashi T, Kosaka H, Wada Y, Yoshida H. Abnormal nocturnal blood pressure fall in senile-onset depression with subcortical silent cerebral infarction. Neuropsychobiology 2003; 47: 187-191.

27 Luckhaus C, Flüb MO, Wittsack HJ, Grass-Kapanke B, Jänner M, Khalili-Amiri R, Friedrich W, Supprian T, Gaebel W, Mödder U, Cohnen M. Detection of changed regional cerebral blood flow in mild cognitive impairment and early Alzheimer's dementia by perfusion-weighted magnetic resonance imaging. Neuroimage 2008; 40: 495-503.

28 Caroli A, Testa C, Geroldi C, Nobili F, Barnden LR, Guerra UP, Bonetti M, Frisoni GB. Cerebral perfusion correlates of conversion to Alzheimer's disease in amnestic mild cognitive impairment. J Neurol 2007; 254: 1698-1707.

29 Sander D, Winbeck K, Klingelhöfer J, Conrad B. Extent of cerebral white matter lesions is related to changes of circadian blood pressure rhythmicity. Arch Neurol 2000; 57: 1302-1307.

30 Kario K, Matsuo T, Kobayashi H, Imiya M, Matsuo M, Shimada K. Nocturnal fall of blood pressure and silent cerebrovascular damage in elderly hypertensive patients. Advanced silent cerebrovascular damage in extreme dippers. Hypertension 1996; 27. 130-135.

31 Smith EE, Egorova S, Blacker D, Killiany RJ, Muzikansky A, Dickerson BC, Tanzi RE, Albert MS, Greenberg SM, Guttmann CR. Magnetic resonance imaging white matter hyperintensities and brain volume in the prediction of mild cognitive impairment and dementia. Arch Neurol 2008; 65: 94-100.

32 Lopez OL, Jagust WJ, Dulberg C, Becker JT, DeKosky ST, Fitzpatrick A, Breitner J, Lyketsos C, Jones B, Kawas C, Carlson M, Kuller LH. Risk factors for mild cognitive impairment in the Cardiovascular Health Study Cognition Study: part 2. Arch Neurol 2003. 60: 1394-1399.

33 Zanetti M, Ballabio C, Abbate C, Cutaia C, Vergani C, Bergamaschini L. Mild cognitive impairment subtypes and vascular dementia in community-dwelling elderly people: a 3-year follow-up study. J Am Geriatr Soc 2006; 54: 580-586.

34 Cicconetti P, Morelli S, Ottaviani L, Chiarotti F, De Serra C, De Marzio P, Costarella M, Sgreccia A, Ciotti V, Marigliano V. Blunted nocturnal fall in blood pressure and left ventricular mass in elderly individuals with recently diagnosed isolated systhypertension. Am J Hypertens 2003; 16: 900-905

35 Moretti R, Torre P, Antonello RM, Manganaro D, Vilotti C, Pizzolato G. Risk factors for vascular dementia: hypotension as a key point. Vasc Health Risk Manag 2008; 4: 395-402.

36 Grassi G, Seravalle G, Quarti-Trevano F, Dell'Oro R, Bombelli M, Cuspidi C, Facchetti R, Bolla G, Mancia G. Adrenergic, metabolic, and reflex abnormalities in reverse and extreme dipper hypertensives. Hypertension 2008; 52: 925-931.

37 Takakuwa H, Shimizu K, Izumiya Y, Kato T, Nakaya I, Yokoyama H, Kobayashi K, Ise T. Dietary sodium restriction restores nocturnal reduction of blood pressure in patients with primary aldosteronism. Hypertens Res 2002; 25: 737-742.

38 Uzu T, Takeji M, Yamauchi A, Kimura G. Circadian rhythm and postural change in natriuresis in non-dipper type of essential hypertension. J Hum Hypertens 2001; 15: 323-327

39 Higashi Y, Oshima T, Ozono R, Nakano Y, Matsuura H, Kambe M, Kajiyama G. Nocturnal decline in blood pressure is attenuated by $\mathrm{NaCl}$ loading in salt-sensitive patients with essential hypertension: noninvasive 24-h ambulatory blood pressure monitoring. Hypertension 1997; 30: 163-167.

40 Kario K, Eguchi K, Nakagawa Y, Motai K, Shimada K. Relationship between extreme dippers and orthostatic hypertension in elderly hypertensive patients. Hypertension 1998; 31: 77-82.

41 Kidd PM. Alzheimer's disease, amnestic mild cognitive impairment, and age-associated memory impairment. Altern Med Rev 2008; 13: 85-115. 